\title{
OPTIMIZATION OF THE REQUIRED TEMPERATURE OF CONTACT SOLDERING OF ELECTRONIC DEVICES
}

\section{N. Shtennikov and V. D. Seleznev}

UDC $621.372 ; 621.791 .3$

This paper presents the results of a complex of experimental and theoretical studies aimed at improving the quality of soldering electronic devices by optimizing the design-technological parameters.

Keywords: engineering physics, devices, soldering, temperature, heat conduction, time.

Introduction. The development of the semiconductor industry and new materials and technologies has opened up prospects for miniaturizing electronic devices and increasing their functionality and reliability but at the same time has aggravated problems connected with their manufacture (Fig. 1).

Experience in production of electronic devices shows that the quality of their manufacture depends primarily on the quality of the soldering operation. Among the factors influencing the quality of soldered joints (soldering time, quality of joined surfaces, size of the gap between them, used flux, solder composition), the soldering temperature is deciding [1].

From experience it follows that the required soldering temperature does not coincide, as a rule, with the temperature set on the equipment (heater, soldering rod) and the ambient temperature. The nonstationary temperature field arises during soldering due to the nonequilibrium processes of heat conduction, heat emission, and convective heat exchange with the environment [2]. Therefore, the soldering temperature depends primarily on the geometrical and temporal conditions of the heat transfer processes. The required quality of soldered joints can be attained by optimal variation of these conditions. However, the large number of parameters influencing the processes under consideration makes the optimization problem difficult to solve [3, 4]. Moreover, printed circuit boards should be soldered, where possible, under unified technological conditions, which follows from the condition of carrying out group soldering, as well as from the necessity of controlling the solderer's work in the process of manual single soldering. The above factors, in the aggregate, complicate considerably the problem to be solved.

Thus, the topicality of the problem to be solved is explained by

1) the necessity of making high-quality electronic devices in the absence of sufficient experience and necessary normative documentations;

2) the strong influence of the quality of soldered joints on the reliability of electronic devices on the whole;

3 ) the large number and diversity of structures of soldered joints in electronic devices requiring original equipment and various soldering technologies;

4) the existence of complex dependences of technological process parameters on equipment parameters;

5) the presence of discrepancies in the technical literature and normative documentation on the given matter, including the international standards IPC and MEC;

6) the existence of only general recommendations on the development and observation of optimal regimes of soldering, the reliability forecast, and the solution of problems of their provision.

Methods of Investigations and Results. The temperature was measured by a chromel-copel thermocouple from wires of diameter $0.2 \mathrm{~mm}$ or a copper-constantan thermocouple from wires of diameter $0.06 \mathrm{~mm}$. In this process, the condition under which the diameter of thermocouple wires was much smaller than the diameter of the measured wire or the soldering rod was satisfied. To record the thermal e.m.f., a KSP-4 potentiometer of accuracy class 0.25 was used. The temperature measurement error was $\pm 1^{\circ} \mathrm{C}$. The soldering time $0.3-5.0 \mathrm{~s}$ was determined with the use of an STTs 1 timer stop-watch.

The temperature measurement error in soldering during $2 \mathrm{~s}$ by a thermocouple located at a distance of $0.5 \mathrm{~mm}$ from the heated or cooled surface was $\pm 2 \%$.

B. N. Yeltsin Ural Federal University, 19 Mir Str., Ekaterinburg, 620002, Russia; email: Shtennikov_vn@mail.ru. Translated from Inzhenerno-Fizicheskii Zhurnal, Vol. 86, No. 5, pp. 997-1001, September-October, 2013. Original article submitted March 25, 2013. 


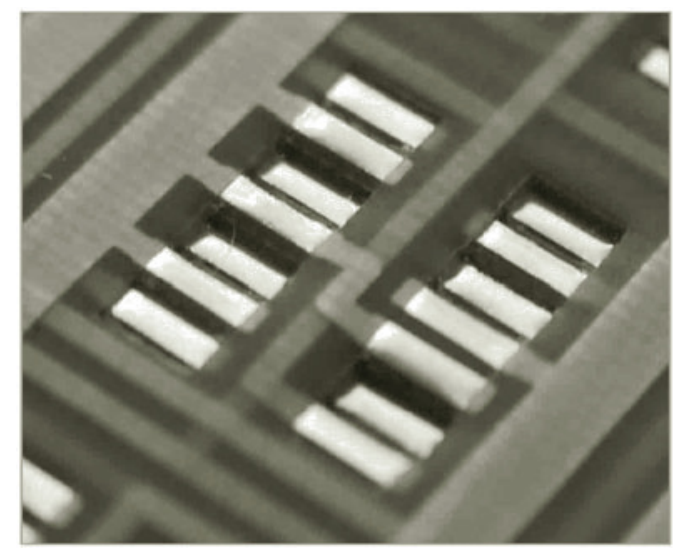

Fig. 1. Multilayer printed circuit board with open terminal pads.

The quality of soldered joints was assessed visually with the use of an MBS-2 microscope with a magnification of $(25-50)^{\mathrm{X}}$; by the method of investigation of the forms and microsections of soldered joints; by measuring the mechanical strength, including measurements on a type 2092 tearing machine with the use of a P772.002 with a value of the scale division of 0.5 newton.

In the approximation of constancy of the heat conductivity coefficient $\lambda$ of the rod material and the heat transfer coefficient $\alpha$ between the soldering rod surface and air, we obtained the expression for the temperature drop $\Delta t_{\text {tip } L}$ along the length of the soldering rod $L$ of constant diameter $d$ when running idle:

$$
\Delta t_{\text {tip }}=t_{\text {tip }}-t_{L} \approx t_{L} L^{2} \frac{2 \alpha}{\lambda d} .
$$

Moreover, we obtained the expression for calculating the Manco efficiency of the solderer as the ratio between the quantity of heat input to the tip and the quantity of heat released by the heating element:

$$
(\text { Efficiency })_{\mathrm{M}}=\frac{\pi}{4} \frac{\alpha d L t_{\text {tip }}}{P_{x x}},
$$

where $P_{x x}$ is the power of the solderer and $t_{\text {tip }}$ is the temperature of the soldering tip of the solderer rod.

Moreover, we obtained the formula for determining the efficiency of the solderer as a ratio between the heat absorption $P_{1}$ (absorption of heat transferred by the soldered joint) and the solderer power during soldering:

$$
(\text { Efficiency })_{\text {sol }}=P_{1} /\left(P_{x x}+P_{1}\right)
$$

On the assumption of constancy of the heat transfer coefficient between the solderer surface and air and nonuniformity of the temperature field in the volume of the solderer, we obtained formulas that permit estimating fairly correctly its average temperature $t_{1}$ depending on the power $D$, the surface area $S_{\mathrm{S}}$, the heat capacity $C_{\mathrm{s}}$, and the heat absorption during soldering:

$$
t=t_{x x}\left(1-e^{-\tau / \tau_{0}}\right)
$$

where $t_{x x}=\frac{P}{\alpha S_{\mathrm{s}}}$ is the solderer temperature at long intervals between acts of soldering, and $\tau_{0}=\frac{C_{\mathrm{s}}}{\alpha S_{\mathrm{s}}}$ is the heating time constant.

Since one of the heat exchange components connected with radiation is a fortiori temperature-dependent, the obtained results describe the process under consideration only qualitatively.

It has been shown theoretically on the basis of comparing the cooling time constants of the soldering rod when in air and in contact with a soldered joint that neglecting the heat exchange of the soldering rod with the air introduces an error of 


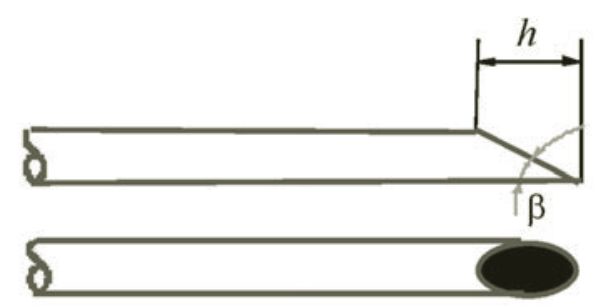

Fig. 2. Sharpened part of a round rod at an angle $\beta=30-45^{\circ}$.

over $1 \%$ in describing the change in the temperature of its soldering tip when in contact with a soldered joint, which is several times lower than the experimental error.

In the approximation of the absence of heat exchange with the air of the soldering rod and a wire of constant crosssectional area, the following equations have been obtained:

$$
\begin{gathered}
\Delta t_{1}=t_{\mathrm{s} 1} \frac{\mathrm{S}_{2} \sqrt{(\lambda \rho c)_{2}}}{\mathrm{~S}_{1} \sqrt{(\lambda \rho c)_{1}}} \\
\Delta t_{1(x, \tau)}=\Delta t_{1}\left\{1-\Phi\left(\frac{x}{2 \sqrt{a_{1} \tau_{\mathrm{s}}}}\right)\right\},
\end{gathered}
$$

where $\Delta t_{1}$ is the decrease in the temperature of the soldering tip of the rod; $S_{2}$ and $S_{1}$ are the cross-sectional areas of the wire and the rod, respectively; $\sqrt{(\lambda \rho c)_{2}}$ and $\sqrt{(\lambda \rho c)_{1}}$ are the heat assimilation coefficients of the wire and the soldering rod material, respectively; $\Phi(u)=\frac{2}{\sqrt{\pi}} \int_{0}^{u} e^{-\xi^{2}} d \xi$ is the probability integral; $a_{1}=\frac{\lambda}{\rho c}$ is the thermal diffusivity of the soldering rod material; and $\tau_{\mathrm{s}}$ is the soldering time.

The agreement, within the limit of experimental error (about 6\%), between the experimental data and the calculated values confirmed the adequacy of the chosen model to the investigated processes.

Using the formula for the temperature gradient on the surface of a semibounded body provided $\delta_{\mathrm{pr}}<<\sqrt{a_{\mathrm{c}} \tau_{\mathrm{s}}}$, we obtained the expression for the decrease in the temperature of the soldering tip of the rod with a protective coat layer of thickness $\delta_{\text {pr }}$ and without it with respect to the decrease in the temperature of the soldering tip of the pure copper rod [5]:

$$
\frac{\Delta t_{\delta \mathrm{pr}}-\Delta t_{\delta \mathrm{c}}}{\Delta t_{1 \mathrm{c}}}=\frac{\delta_{\mathrm{pr}}}{\sqrt{\pi a_{\mathrm{c}} \tau_{\mathrm{s}}}}\left(\frac{\lambda_{\mathrm{c}}}{\lambda_{\delta}}-1\right)
$$

According to this expression, at a soldering time of $1 \mathrm{~s}$ and a thickness of the iron coat of $0.13 \mathrm{~mm}$ and of the nickel coat of $0.18 \mathrm{~mm}$, the decrease in the temperature of the soldering tip of the rod during soldering will amount to $105 \%$ as compared to the pure copper rod. In the limit of experimental error, the theoretical and experimental data agree.

Fulfilling the condition $h<<\sqrt{a_{\mathrm{c}} \tau_{\mathrm{s}}}$, we obtained the expression for the temperature decrease along the sharpened length $h$ of the soldering rod with respect to the decrease in the temperature of an unsharpened soldering rod of length $h$ (Fig. 2):

$$
\frac{\Delta t_{h \mathrm{pr}}-\Delta t_{h \mathrm{c}}}{\Delta t_{\mathrm{lc}}}=\frac{h}{\sqrt{\pi a_{\mathrm{c}} \tau_{\mathrm{s}}}}\left(\frac{R_{\mathrm{c}}}{R_{h}}-1\right),
$$

where $R_{\mathrm{c}}$ and $R_{h}$ are the heat resistance of a rod of length $h$ without sharpening and with sharpening, respectively.

The obtained relation shows that the presence of a sharpened part of the soldering tip of the rod at an angle of $30^{\circ}$ decreases the soldering temperature of the leads of electronic radio devices onto open terminal pads by $7-15^{\circ} \mathrm{C}$ at a soldering time of $2 \mathrm{~s}$ for a rod of diameter $2 \mathrm{~mm}$.

Expanding the function of errors in formula (6) into a series at small argument values, we obtained the expression for estimating the temperature drop $\Delta t_{1}$ along the lead or wire length $x$ with respect to their interface with the soldering rod [6]: 


$$
\Delta t_{1}=\frac{x t_{\mathrm{s}}}{\left.\sqrt{\left(\pi a_{1}\right.} \tau_{\mathrm{s}}\right)},
$$

where $\Delta t_{\mathrm{S}}$ is the soldering temperature.

Thus, to provide high-quality soldering of component leads into the holes of a printed circuit board, it is necessary to increase the soldering temperature on the side of solder feed by a value proportional to the thickness of the printed circuit board and inversely proportional to the square root of the soldering time.

Relations (8) and (9) agree well with our experimental data and permit formulating additionally the following recommendations on the step soldering and the soldering of thermosensitive components, respectively: it is necessary to increase the distance between soldered joints (between the soldered joint and the thermosensitive component); one can use male contacts (leads), for example, from steel tinned wire or from Kovar instead of copper, and here it is possible to solder a second joint, due to the decrease in the thermal diffusivity, at a distance several times shorter than with the use of a copper lead; it is necessary to shorten the soldering time, having provided the formation of the required diffusion layer and, consequently, the strength of the soldered joint due to preliminary tinning under standard conditions; it is necessary to use a heat sink with the maximum cross-sectional area and the maximum area of actual contact with the lead, which can be achieved, for example, by introducing a liquid or soft metal, a flux; the heat sink should be located as close to the first soldered joint on the side of the second one as possible (as close to the thermosensitive component as possible); locating the heat sink near the second soldered joint will lead to the necessity of increasing the idle run temperature and (or) the diameter of the soldering rod in order to maintain the soldering temperature and thus decrease the heat removal efficiency.

As a result of the numerical solution of the known equation of cooling a bounded plate, one of whose surfaces is isothermal and the other one is exposed to a heat flow whose action is equivalent to the action of a soldered joint, namely of the form const $\sqrt{\tau_{\mathrm{s}}}$ [7], we obtained the formula for a short soldering $\operatorname{rod} L \leq 1.6 \sqrt{a_{1} \tau_{\mathrm{s}}}$ with an isothermal nonsoldering tip:

$$
\frac{\Delta t_{1 \mathrm{is}}}{\Delta t_{1}} \approx 0.62 \mathrm{Fo}^{-0.5}
$$

where $t_{1 \text { is }}$ is the temperature of the soldering tip of a short rod during soldering, and Fo $\equiv \frac{a \tau}{L^{2}}$ is a Fourier criterion.

Using the known solution for heating a bounded plate, one of whose surfaces is isothermal and the other one is heatinsulated [7], we obtained the expression for the heating time constant $\tau_{\text {is }}$ of the soldering tip of a short rod with an isothermal nonsoldering tip:

$$
\tau_{0 \text { is }}=\frac{4 L^{2}}{\pi^{2} a_{1}} .
$$

As follows from Eq. (11), the heating time of the soldering tip of a short rod of soldering equipment after single soldering does not depend on its diameter, which agrees well with the experiment. It has been shown theoretically that the replacement of copper by silver in using a short soldering rod with an isothermal nonsoldering tip somewhat increases the stability of the soldering temperature due to the higher heat conductivity coefficient of silver. The application of short soldering rods from silver permits decreasing also the heating time of the soldering tip after soldering due to the higher heat conductivity coefficient by a factor of about $1.5[8]$.

The established dependence of the temperature of the soldering tip of the rod on its length and the soldering time permits adopting a semi-automatic method of wiring integrated circuits of multilayer printed circuit boards with open terminal pads (MPCB with OTP) of a number of electronic devices, including high-speed soldering with a time of $0.5 \mathrm{~s}$ instead of the traditional time of $2-3 \mathrm{~s}$.

The values of the mechanical strength of joints soldered with account for our recommendations are not lower than the strength of manually soldered joints, and the requirements of OSR4 G0 054.014 "Units and assemblies of IC radio electronic equipment. TTP of assembly" are fulfilled thereby with considerable reserve (8 newtons per lead instead of 1 newton per lead).

The high quality of soldering has been confirmed by a complex of tests of units of automated equipment for resistance to the action of mechanical leads, tests of devices by experimental storage and for reliability, by testing the mechanical strength of a few thousands of soldered joints, by visual control of a few dozens of thousands of soldered joints, by the 
investigations of metallographic specimens and forms of soldered joints, and by the investigation of the breakage boundaries of soldered joints.

Conclusions. We have established a complex of analytical relations between the parameters of the technological process, the equipment, and the structure of soldered joints, which have made it possible to formulate the corresponding proposals and recommendations, enter them into the normative documentation, and bring them into full-scale production, in particular:

1) it has been shown that the decrease in the temperature of the solderer and its heating time depend on its power, heat capacity, and heat absorption during soldering;

2) it has been established that the initial temperature of the soldering rod, its diameter and length, the conditions of its thermal contact with the solderer housing, the protective coat, the sharpening form, and the heat assimilation coefficient influence the temperature of its working tip during soldering;

3 ) it has been determined that the size and the thermal diffusivity of the soldering rod material, the soldering time, and the conditions of thermal contact with the housing of the solderer influence the heating time of the working tip of the rod after soldering;

4) it has been established that to improve the quality of step soldering, it is necessary to use eutectic solders, a heat sink with the maximum actual contact area located near the first soldered joint, shorten the soldering time, increase the distance between soldered joints, and use contacts from a material with the lowest possible heat conductivity coefficient.

It has been shown that in mounting components in the holes of the printed circuit board, it is necessary to take into account the temperature drop in the thickness of the printed circuit board depending on the thickness of the printed circuit board, the soldering time, the material of mounted components, and the presence of a heat sink or a wide printed conductor on the side of the component; the size of the soldered joint regulates the minimum time of contact soldering because of the inadmissibly high temperature drop in its depth.

\section{NOTATION}

$a_{1}=\frac{\lambda}{\rho c}$, thermal diffusivity of the soldering rod material, $\mathrm{m}^{2} \cdot \mathrm{s}^{-1} ; C_{\mathrm{s}}$, heat capacity of the soldering tool, $\mathrm{J} \cdot \mathrm{s}^{-1} ; d$, diameter of the soldering rod, $\mathrm{m} ; \mathrm{Fo} \equiv \frac{a \tau}{L^{2}}$, Fourier criterion; $h$, length of the sharpened part of the soldering rod, $\mathrm{m} ; P_{1}$, heat absorption (power expended in heating soldered joints), W; $P_{x x}$, power of the solderer tool, $\mathrm{W} ; R_{h}$, heat resistance of a rod of length $h$ without sharpening and with sharpening, respectively, ${ }^{\circ} \mathrm{C} / \mathrm{W} ; S_{2}$ and $S_{1}$, cross-sectional area of the wire and the rod, respectively; $S_{\mathrm{s}}$, surface area of the solderer tool, $\mathrm{m}^{2} ; t_{\text {tip }}$, temperature of the tip of the of the solderer, ${ }^{\circ} \mathrm{C} ; t_{x x}=\frac{P}{\alpha S_{\mathrm{s}}}$, temperature of the solderer tool at long intervals between soldering acts, ${ }^{\circ} \mathrm{C} ; t_{1 \text { sol }}$, temperature of the soldering tip of a short rod during soldering, ${ }^{\circ} \mathrm{C} ; x$, distance from the interface between the soldering rod and the wire, $\mathrm{m} ; \Phi(u)=\frac{2}{\sqrt{\pi}} \int_{0}^{u} e^{-\xi^{2}} d \xi$, probability integral; $\alpha$, heat transfer coefficient between the soldering rod surface and the air, $\mathrm{W} \cdot{ }^{\circ} \mathrm{C}^{-1} \cdot \mathrm{m}^{-2} ; \delta_{\mathrm{pr}}$, thickness of the protective coat of the soldering rod; subscripts "pr" and "c" pertain to the protective coat and copper, respectively, $\mathrm{m}^{2} ; \Delta t_{1}$, decrease in the temperature of the soldering tip of the rod, ${ }^{\circ} \mathrm{C} ; \Delta t_{1}$, temperature drop along the length of the lead or the wire, ${ }^{\circ} \mathrm{C} ; \Delta t_{\text {tipL } L}$, temperature drop along the length of the soldering $\operatorname{rod},{ }^{\circ} \mathrm{C} ; \Delta t_{\mathrm{s}}$, soldering temperature, ${ }^{\circ} \mathrm{C} ; \lambda$, heat conductivity coefficient, $\mathrm{W} \cdot \mathrm{m}^{-1} \cdot{ }^{\mathrm{o}} \mathrm{C} ; \tau_{0}=\frac{C_{\mathrm{s}}}{\alpha S_{\mathrm{s}}}$, heating time constant of the solderer, $\mathrm{s} ; \tau_{0 \text { sol }}$, heating time constant of the soldering tip of a short rod with an isothermal soldering tip, $\mathrm{s} ; \tau_{\mathrm{s}}$, soldering time, $\mathrm{s} ; \sqrt{(\lambda \rho c)_{2}}$ and $\sqrt{(\lambda \rho c)_{1}}$, heat assimilation coefficient of the wire and soldering rod materials, respectively, $\mathrm{J} \cdot \mathrm{s}^{-1 / 2}$. Subscripts: 1, lead; pr, protective; c, copper; s, soldering; is, isothermal; sol, solderer; tip, tip.

\section{REFERENCES}

1. N. F. Lashko and S. V. Lashko, Soldering of Metals [in Russian], $2^{\text {nd }}$ edn., Mashinostroenie, Moscow (1967).

2. V. N. Skokov and V. D. Seleznev, Introduction to the Physics of Nonequilibrium Processes [in Russian], Izd. Ural'sk. Gos. Tekh. Univ. — Ural'sk. Politekh. Inst., Ekaterinburg (2010).

3. A. V. Luikov, Heat and Mass Transfer: Handbook [in Russian], $2^{\text {nd }}$ edn., Énergiya, Moscow (1978). 
4. S. A. Zhdanok, O. G. Penyazkov, and N. A. Fomin, Development of R. I. Soloukhin's scientific line of investigations at the Heat and Mass Transfer Institute, Inzh.-Fiz. Zh., 83, No. 6, 1041-1057 (2010).

5. V. N. Shtennikov, International IPC standards for soldering steel rods, Nano- Mikrosist. Tekh., No. 10, $28-29$ (2009).

6. V. N. Shtennikov, Some characteristic features of contact and laser soldering, Pribory, No. 2, 61-63 (2010).

7. A. I. Pekhovich and V. M. Zhidkikh, Calculations of the Thermal Regime of Solid Bodies [in Russian], Énergiya, Leningrad (1976).

8. Thermal Diffusivity of Certain Materials. Reference information. http://temperatures.ru/pages/temperaturoprovodnost_ nekotorykh_materialov 